\title{
FLUX LIMITATION IN ULTRAFILTRATION: OSMOTIC PRESSURE MODEL AND GEL LAYER MODEL
}

\author{
J.G. WIJMANS, S. NAKAO* and C.A. SMOLDERS \\ Department of Chemical Technology, Twente University of Technology, P.O. Box 217, \\ 7500 AE Enschede (The Netherlands)
}

(Received November 28, 1983; accepted in revised form February 22, 1984)

\section{Summary}

The characteristic permeate flux behaviour in ultrafiltration, i.e., the existence of a limiting flux which is independent of applied pressure and membrane resistance and a linear plot of the limiting flux versus the logarithm of the feed concentration, is explained by the osmotic pressure model. In the mathematical description presented here, a quantity $\Delta \Pi n /\left(R_{\mathrm{m}} k\right)$ is introduced which is the ratio of the resistance caused by the osmotic pressure and the resistance of the membrane itself. For high values of this quantity $(>19)$ the flux is practically limited by the osmotic pressure.

Factors leading to high values of the quantity $\Delta \Pi n /\left(R_{\mathrm{m}} k\right)$ are discussed and it is concluded that in the ultrafiltration of medium molecular weight solutes $(10,000$ to 100,000 daltons) osmotic pressure limitation is more likely than gel layer limitation.

\section{Introduction}

Ultrafiltration is a pressure-driven membrane process by which macromolecular solutes are separated from the solvent. In actual applications the permeate flux increases less than linearly with the pressure difference over the membrane, and it is always smaller than the pure solvent flux. At higher pressure differences the permeate flux is no longer significantly affected by the pressure difference; it levels off to almost constant values. This constant flux is called "limiting flux" and is independent of membrane resistance. Under unchanged mass transfer conditions only the feed concentration is an important variable, and a linear relationship can be obtained in the plot of the limiting flux versus the logarithm of the feed concentration.

These characteristic phenomena have been explained by the gel layer model $[1,2]$. In addition to this model another mechanism for flux limitation, the socalled osmotic pressure model, has been proposed [3,4]. Since the osmotic pressure model has received more and more attention recently $[5,6]$, it is very interesting to investigate whether this model is capable of predicting the complete flux behaviour. This is the aim of the present work.

*Present address; Institute of Industrial Science, University of Tokyo, 7-22-1 Roppongi, Minatoku, Tokyo 106, Japan. 


\section{The gel layer model $[1,2]$}

The concentration of the solute at the membrane surface, $c_{\mathrm{m}}$, is much higher than that in the bulk solution due to concentration polarization. Assuming a rejection of $100 \%$ and neglecting the influence of concentration profile and permeate flux on the mass transfer coefficient, $k$, the following relation for $c_{m}$ is obtained [2]:

$c_{\mathrm{m}}=c_{\mathrm{b}} \exp (J / k)$

where $c_{b}$ is the bulk concentration and $J$ is the permeate flux. The concentration $c_{\mathrm{m}}$ increases rapidly with the permeate flux, reaching a concentration $c_{\mathrm{g}}$ where the solution is no longer fluid. The thus formed gel layer at the membrane surface has a hydraulic resistance which reduces the permeate flux to such an extent that

$J_{\infty}=k \ln \left(c_{\mathrm{g}} / c_{\mathrm{b}}\right)$

where $J_{\infty}$ is the limiting flux in ultrafiltration. Since the gel concentration is assumed to be constant, eqn. (2) predicts a linear plot for $J_{\infty}$ versus $\ln c_{\mathrm{b}}$ with a slope equal to $-k$; extrapolation to $J_{\infty}=0$ will yield the $\ln c_{\mathrm{g}}$ value. It also predicts that $J_{\infty}$ is not a function of the membrane resistance as long as the rejection value is constant.

In practice it appears that eqn. (2) is very useful in correlating experimental limiting fluxes $[2,7]$, but it has also been shown that the information obtained on gel concentrations is not reliable. For identical solutions different authors find widely varying values for $c_{\mathbf{g}}$ [8], and these values are sometimes too low (solutions at that concentration being still fluid), sometimes too high ( $c_{\mathrm{g}}$ values found by extrapolation exceeding $100 \%$ ).

\section{The osmotic pressure model $[3,4]$}

The concentration $c_{\mathrm{m}}$ is significantly higher than the bulk concentration and therefore the osmotic pressure of the solution at the membrane surface is no longer negligible. The permeate flux is governed in this case by:

$J=\frac{\Delta P-\Delta \Pi}{R_{\mathrm{m}}}$

where $\Delta P$ is the hydraulic pressure difference, $\Delta \Pi$ is the osmotic pressure difference and $R_{\mathrm{m}}$ is the membrane resistance. Again a rejection of $100 \%$ is assumed and in that case the osmotic pressure difference is determined by the concentration at the membrane surface, $c_{m}$.

When the applied pressure is increased the permeate flux will at first increase. This results in a higher value for $c_{\mathrm{m}}$ and thus in a larger osmotic pressure. In this way the pressure increase is partly cancelled by the osmotic pressure increase. If $\Delta \Pi$ increases rapidly with the permeate flux, the increase in $\Delta P$ may lead to only a small increase in the permeate flux. 


\section{Mathematical description of the osmotic pressure model}

In this section the mathematical expressions which show the characteristic flux behaviour are derived. The osmotic pressure difference of macromolecular solutions relative to the pure solvent increases much more than linearly with concentration, which can be represented as follows:

$\Delta \Pi=a c^{n}$

where $a$ is a constant and $n$ an exponent larger than 1. De Gennes [9] has shown that for semidilute macromolecular solutions $n$ will have a value of about two. For more concentrated solutions the exponent is even larger than two [10].

The osmotic pressure difference, $\Delta \Pi$, strongly depends on the permeate flux, $J$, since the latter determines the concentration $c_{\mathrm{m}}$, as is shown by eqn. (1). Thus eqn. (3) can be rewritten as:

$J=\frac{\Delta P-a c_{\mathrm{b}}^{n} \exp (n J / k)}{R_{\mathrm{m}}}$

From eqn. (5) it is clear that $J$ will not increase linearly with $\Delta P$. For the derivative $\partial J / \partial \Delta P$ we find:

$$
\begin{aligned}
\frac{\partial J}{\partial \Delta P} & =\left[R_{\mathrm{m}}+a c_{\mathrm{b}}^{n}(n / k) \exp (n J / k)\right]^{-1} \\
& =\left(R_{\mathrm{m}}+(n / k) \Delta \Pi\right)^{-1} \\
& =\frac{1}{R_{\mathrm{m}}}\left(1+\frac{\Delta \Pi n}{R_{\mathrm{m}} k}\right)^{-1}
\end{aligned}
$$

and we see that for high effective osmotic pressures the increase in $J$ with $\Delta P$ is almost zero.

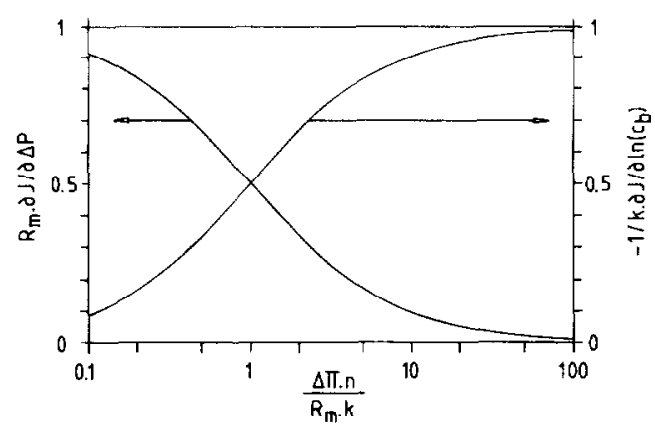

Fig. 1. Effectiveness of pressure increase, $R_{\mathrm{m}} \partial J / \partial \Delta P$, versus the ratio of osmotic resistance and membrane resistance, $\Delta \Pi n /\left(R_{\mathrm{m}} k\right)$. Derivative of permeate flux with respect to the logarithm of bulk concentration, divided by the mass transfer coefficient, $-1 / k\left(\partial J / \partial \ln c_{b}\right)$, versus the ratio $\Delta \mathrm{II} n /\left(R_{\mathrm{m}} k\right)$. 
In Fig. 1 the quantity $R_{m}(\partial J / \partial \Delta P)$ is represented as a function of the quantity $\Delta \Pi n /\left(R_{\mathrm{m}} k\right)$. The physical meaning of these two dimensionless numbers is illustrated by the following equations which can both be derived from eqn. (5):

$R_{\mathrm{m}} \frac{\partial J}{\partial \Delta P}=\frac{\partial J}{\partial \Delta P} /\left(\frac{\partial J}{\partial \Delta P}\right)_{\text {pure solvent }}$

and

$\frac{\Delta \Pi n}{R_{\mathrm{m}} k}=\frac{\partial \Delta \Pi}{\partial J} / R_{\mathrm{m}}$

Thus, $R_{\mathrm{m}}(\partial J / \partial \Delta P)$ is the ratio of the slope of the actual $J$ versus $\Delta P$ curve and that of the pure solvent flux $J_{\text {ps }}$ versus $\Delta P$ curve. The latter slope is the maximum slope which can be achieved, so $R_{\mathrm{m}}(\partial J / \partial \Delta P)$ is a measure for the effectiveness of a pressure increase. $\Delta \Pi n /\left(R_{\mathrm{m}} k\right)$ appears to be the ratio of the resistance caused by the osmotic pressure and the resistance of the membrane itself. The sum of these two resistances constitutes the actual total resistance, as is deduced from eqn. (3):

$\frac{\partial \Delta P}{\partial J}=R_{\mathrm{m}}+\frac{\partial \Delta \Pi}{\partial J}$

Figure 1 shows that the build up of an osmotic pressure gradually leads to a limiting flux. The effectiveness of a pressure increase becomes less and one can define more or less arbitrarily a "limiting flux region". If the permeate flux is supposed to be a limiting flux when $\partial J / \partial \Delta P$ is $5 \%$ or less of the pure solvent permeability, then the condition for flux limitation is from Fig. 1 seen to be:

$\frac{\Delta \Pi n}{R_{\mathrm{m}} k}>19$

The second important feature, i.e., the permeate flux as a function of bulk concentration, can also be described by a derivative. After rearrangement and differentiation of eqn. (5), one obtains:

$\frac{\partial J}{\partial \ln \left(c_{\mathrm{b}}\right)}=-\left(\frac{1}{k}+\frac{1 / n}{\Delta P / R_{\mathrm{m}}-J}\right)^{-1}$

$$
\begin{aligned}
& =-k\left(1+\frac{1 / n}{\operatorname{Pe}\left(J_{\mathrm{ps}} / J-1\right)}\right)^{-1} \\
& =-k\left(1+\frac{R_{\mathrm{m}} k}{\Delta \Pi n}\right)^{-1}
\end{aligned}
$$

where $P e=J / k$ is the boundary layer Pèclet number and $J_{\mathrm{ps}}=\Delta P / R_{\mathrm{m}}$ is the 
pure solvent flux. Equation (11) shows that a plot of $J$ versus $\ln c_{\mathrm{b}}$ will yield a straight line with a slope equal to $-k$ when $J_{\mathrm{ps}} / J$ is large and/or when the Pèclet number is large. These two conditions can be combined into one: $\Delta \Pi n /\left(R_{\mathrm{m}} k\right) \gg 1$. In Fig. 1 the quantity $\partial J / \partial \ln c_{\mathrm{b}}$ divided by $-k$ is given as a function of $\Delta \Pi n /\left(R_{\mathrm{m}} k\right)$. In the "limiting flux region" the slope of a $J$ versus $\ln c_{\mathrm{b}}$ plot is equal to $-k$ within $5 \%$. It must be noted that the absolute value of the actual slope is always smaller than $k$.

At high values of the ratio $\Delta \Pi n /\left(R_{\mathrm{m}} k\right)$ the permeate flux depends only slightly on the membrane resistance $R_{\mathrm{m}}$. This can be inferred from eqn. (5), presented here in a different way:

$J R_{\mathrm{m}}+a c_{\mathrm{b}}^{n} \exp (n J / k)=\Delta P$

When $\Delta \Pi n /\left(R_{\mathrm{m}} k\right)$ is large, the term $J R_{\mathrm{m}}$ is small compared to $\Delta \Pi$ and $\Delta P$. In this case the permeate flux is given by:

$J=(k / n) \ln \left(\Delta P /\left(a c_{\mathrm{b}}^{n}\right)\right)$

which, upon differentiating with respect to $\ln c_{\mathrm{b}}$, yields a slope exactly equal to $-k$. Equation (12) is also obtained for the highly hypothetical case of a membrane with no hydrodynamic resistance and with a perfect solute rejection.

\section{Calculated example of osmotic pressure effect}

In Figs. 2 and 3 the results of a model calculation, using eqn. (5) are given. The parameters are chosen to be characteristic for a typical ultrafiltration experiment:

$n=2$

$a=100 \mathrm{~atm}$

$R_{\mathrm{m}}=5 \cdot 10^{5} \mathrm{~atm}-\mathrm{sec} / \mathrm{m}$

$k=2 \cdot 10^{-6} \mathrm{~m} / \mathrm{sec}$

$\Delta P=0-10 \mathrm{~atm}$ (a macromolecular solute with $\bar{M}_{\mathrm{n}}=70,000$ )

$(\Delta \Pi=1 \mathrm{~atm}$ at weight fraction of 0.1$)$

(a membrane having a molecular weight cut-off of about 40,000)

(turbulent flow $(R e=5,000)$, diffusion coefficient of solute $=5 \cdot 10^{-11} \mathrm{~m}^{2} / \mathrm{sec}$ )

$c_{\mathrm{b}}=0.0001-0.1$ weight fraction

As is expected the effectiveness of pressure increase becomes gradually less for higher applied pressures and higher bulk concentrations, and the permeate fluxes decrease with increasing bulk concentrations, see Fig. 2. Most commercial ultrafiltration membranes possess a membrane resistance which is less than the value of $5 \cdot 10^{5} \mathrm{~atm}-\mathrm{sec} / \mathrm{m}$ used in our calculation. As will be clear from the analysis given above, lower values for $R_{\mathrm{m}}$ lead to a more pronounced osmotic pressure effect, i.e., flux limitation at lower applied pressures.

In Fig. 3 the permeate flux is plotted as a function of $c_{\mathrm{b}}$ for two different applied pressures. It is clear that for high bulk concentrations, that is, for high values of $\Delta \Pi n /\left(R_{\mathrm{m}} k\right)$, the slope $\partial J / \partial \ln c_{\mathrm{b}}$ approaches $-k$ (see broken lines in 


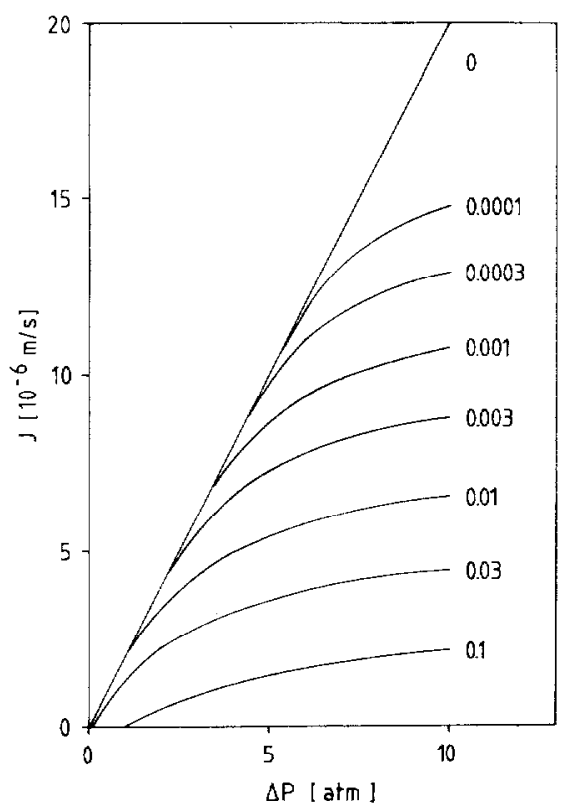

Fig. 2. Calculated permeate flux as a function of applied pressure. Numbers identifying each curve indicate bulk concentration, $c_{\mathrm{b}}$.

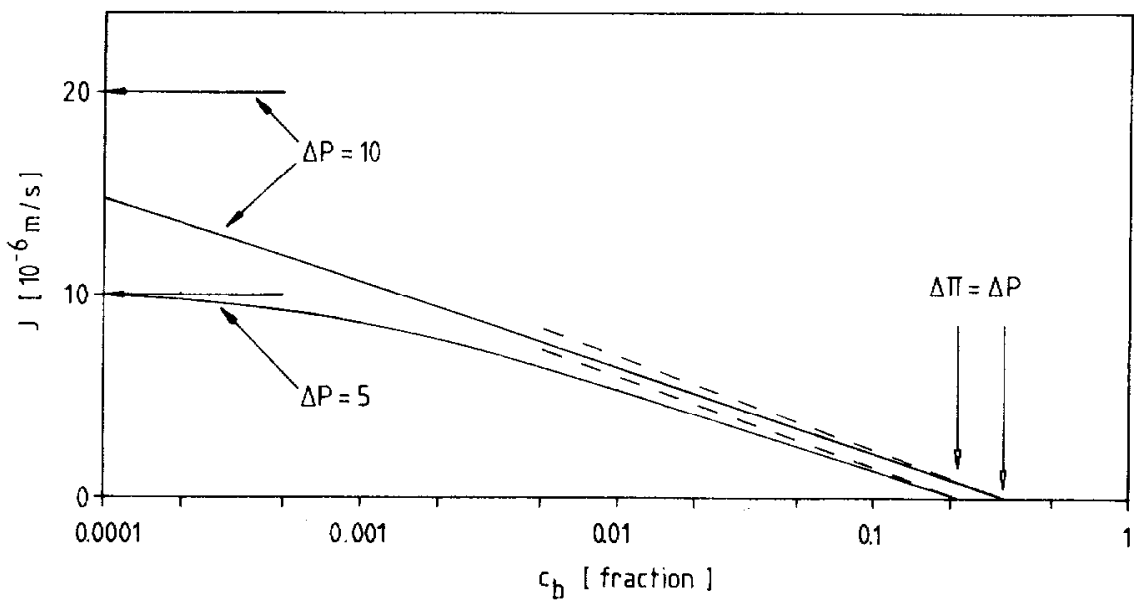

Fig. 3. Permeate flux. $J$, as a function of bulk concentration for two pressures: $\Delta P=\mathbf{5}$ atm and $\Delta P=10 \mathrm{~atm}$. $\leftarrow$ : pure solvent flux; - : ultrafiltration flux; $\cdots \cdot-$ : slope $=-k$.

Fig. 3). For low bulk concentrations $J$ approaches the pure solvent flux. The intercept at the $J=0$ axis gives the bulk concentration for which $\Delta \Pi=\Delta P$. In this respect the osmotic pressure model deviates from the gel layer model, which predicts an intercept not depending on $\Delta P$. 
Figure 4 illustrates the diminishing importance of the membrane resistance, $R_{\mathrm{m}}$, as the osmotic pressure increases. For high osmotic pressures, the actual permeate flux approaches the permeate flux of the "ideal" membrane as described by eqn. (12).

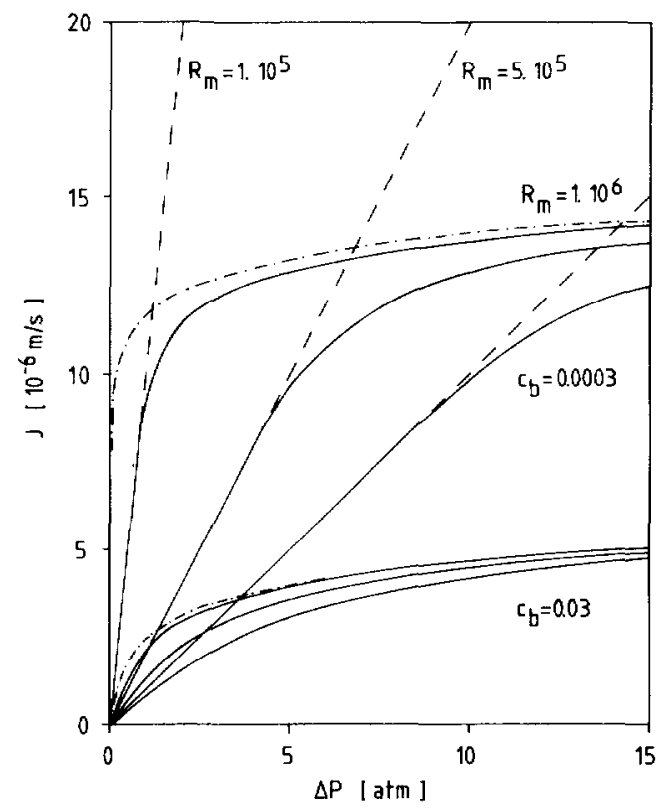

Fig. 4. Permeate flux, $J$, as a function of applied pressure, $\Delta P$, for two different feed concentrations and three different membrane permeabilities. $n=2 ; a=100 \mathrm{~atm} ; k=$ $2.10^{-6} \mathrm{~m} / \mathrm{sec} . \cdot . \cdot$ : pure solvent flux; - : ultrafiltration permeate flux; $\cdots \cdots$ : permeate flux calculated using eqn. (12).

\section{Discussion and conclusions}

It has been shown that the osmotic pressure model is capable of explaining the most important features of the permeate flux behaviour in ultrafiltration. This is confirmed with the model calculation and it can be illustrated by experimental data [4,11]. Goldsmith [4] has shown that under circumstances where no gel layer is expected, i.e., a low molecular weight polyethylene glycol $\left(\bar{M}_{\mathrm{n}}=15,500\right.$ daltons) as the solute and the concentration $c_{\mathrm{m}}$ less than $10 \%$ by weight, an almost limiting flux can be obtained, whereby the $J$ versus $\ln c_{\mathrm{b}}$ plot is linear for a given pressure $\Delta P$. Vilker et al. [11] concluded from osmotic pressure measurements and ultrafiltration experiments that the permeate flux in the ultrafiltration of bovine serum albumine is limited by the osmotic pressure.

The predictions of the osmotic pressure model are practically equivalent to those of the gel layer model. Two differences are: (a) the osmotic pressure 
model does not predict a fully limiting flux, and (b) contrary to the gel layer model, the osmotic pressure model explains the deviation of the permeate flux from the pure solvent flux at low pressures. At high pressure differences, the dependency of the permeate flux on the pressure difference decreases gradually. Eventually this dependency becomes so small that it is hidden within the experimental error.

The analogous result obtained from both models makes it very difficult to conclude from experimental data which mechanism is actually in operation. A possible answer may come from analyzing the intercept at the $J=0$ axis in the $J$ versus $\ln c_{\mathrm{b}}$ plot. According to the two mechanisms discussed, there are two possibilities: (a) the concentration thus found has an osmotic pressure nearly equal to the applied pressure difference, or (b) it is reasonable on physical grounds to designate this concentration as the gel concentration.

It is also possible to examine the probability of osmotic pressure limitation by evaluating the value of the ratio $\Delta \Pi n /\left(R_{\mathrm{m}} k\right)$. In this case data on mass transfer coefficient and osmotic pressure must be available. See Table 1 for values of $\Delta \Pi n /\left(R_{\mathrm{m}} k\right)$ in the model calculation displayed in Fig. 4. We will summarize here the factors which lead to a high value of this ratio:

- a high permeate flux, i.e., a high applied pressure or a low membrane resistance;

- a high feed concentration;

- a low mass transfer coefficient, i.e., a low degree of mixing near the membrane surface or a small diffusion coefficient of the solute;

- a high exponent $n$, i.e., a macromolecular solute;

- a high value of the constant $a$, i.e., a low molecular weight of the solute (which will be counteractive through the diffusion coefficient and the exponent $n$ ).

From these factors it can be concluded that in ultrafiltration using solutes with molecular weights in medium (10,000 to 100,000 daltons) and high $(>100,000$ daltons) ranges, the permeate flux may be limited by osmotic pressure. There are two additional considerations: (a) the concentration $c_{m}$ of a

\section{TABLE 1}

Ratio of osmotic resistance and membrane resistance, $\Delta \Pi n /\left(R_{\mathrm{m}} k\right)$, calculated at $\Delta P=$ $10 \mathrm{~atm}$ for the model calculation displayed in Fig. 4. Reduction of the flux by osmotic pressure is most severe for high flux membranes

\begin{tabular}{lll}
\hline$R_{\mathrm{m}}$ & \multicolumn{2}{c}{$\Delta \Pi n /\left(R_{\mathrm{m}} k\right)$} \\
\cline { 2 - 3 }$\left(10^{5} \mathrm{~atm}-\mathrm{sec} / \mathrm{m}\right)$ & $c_{\mathrm{b}}=0.0003$ & $c_{\mathrm{b}}=0.03$ \\
\hline 0 & $\infty$ & $\infty$ \\
1 & 86.22 & 95.30 \\
5 & 7.12 & 15.54 \\
10 & 0.17 & 6.83 \\
\hline
\end{tabular}


high molecular weight solute must be very high to give effective osmotic pressures; and (b) the gel concentration of a high molecular weight solute will be low. These considerations make gel layer limitation more likely in ultrafiltration using high molecular weight solutes, whereas osmotic pressure limitation is expected in ultrafiltration using medium molecular weight solutes.

\section{Acknowledgement}

The authors are indebted to M.J. van der Waal for stimulating discussions during the preparation of the manuscript.

\section{List of symbols}

$\begin{array}{ll}a & \text { atm } \\ c & \text { weight fraction } \\ c_{\mathrm{b}} & \text { weight fraction } \\ c_{\mathrm{g}} & \text { weight fraction } \\ c_{\mathrm{m}} & \text { weight fraction } \\ J & \mathrm{~m} / \mathrm{sec} \\ J_{\mathrm{ps}} & \mathrm{m} / \mathrm{sec} \\ \frac{k}{M_{\mathrm{n}}} & \mathrm{m} / \mathrm{sec} \\ n & \text { dalton } \\ P e & - \\ R e & - \\ R & \text { atm-sec } / \mathrm{m} \\ \Delta P & \text { atm } \\ \Delta \Pi & \text { atm }\end{array}$

constant defined by eqn. (4)

solute concentration

solute concentration in bulk of feed

gel concentration of solute

solute concentration at membrane surface

ultrafiltration permeate Mux

pure solvent flux

mass transfer coefficient

number averaged molecular weight

exponent defined by eqn. (4)

Pèclet number

Reynolds number

membrane resistance

hydraulic pressure difference

osmotic pressure difference

\section{References}

1 H.J. Bixler, L.M. Nelsen and L.W. Bluemle, Jr., The development of a diafiltration system for blood purification, 'Irans. Amer. Soc. Artif. Intern. Organs, 14 (1968) 99.

2 W.F. Blatt, A. Dravid, A.S. Micheals and L.M. Nelsen, Solute polarization and cake formation in membrane ultrafiltration: causes, consequences, and control techniques, in: J.E. Flinn (Ed.), Membrane Science and Technology, Plenum Press, New York, 1970.

3 A.A. Kozinski and E.N. Lightfoot, Ultrafiltration of proteins in stagnation flow, AIChE J., 17 (1971) 81.

4 R.L. Goldsmith, Macromolecular ultrafiltration with microporous membranes, Ind. Eng. Chem., Fundam., 10 (1971) 113.

5 P. Aptel and M. Clifton, Ultrafiltration, Lecture presented at the NATO Advanced Studies Institute on Synthetic Membranes, Alcabideche, Portugal, 1983.

6 G. Jonsson, Ultrafiltration of highly viscous solutions, Lecture presented at the 4 th Symposium on Synthetic Membranes in Science and Industry, Tübingen, F.R.G., 1983.

7 M.C. Porter, Concentration polarization with membrane ultrafiltration, Ind. Eng. Chem., Prod. Res. Develop., 11 (1972) 234. 
8 P. Dejmek, Concentration polarization in ultrafiltration of macromolecules, Ph.D. Thesis, Lund Institute of Technology, Sweden, 1975.

9 P.-G. de Gennes, Scaling Concepts in Polymer Physics, Cornell University Press, Ithaca, 1979.

10 P.J. Flory, Principles of Polymer Chemistry, Cornell University Press, Ithaca, 1953.

11 V.L. Vilker, C.K. Colton, K.A. Smith and D.L. Green, The osmotic pressure of concentrated protein and lipoprotein solutions and its significance to ultrafiltration, J. Membrane Sci., 20 (1984) 63. 